\title{
Introduction: healthcare financing - an international comparison of models and outcomes
}

\section{Jos Boertjens, Johan van Manen, Misja Mikkers and Wolf Sauter}

\section{OUTLINE OF THE BOOK: GENESIS AND PURPOSE}

Because the risk of ill health is part of the human condition, there is a universal interest in providing access to high-quality healthcare while controlling the sacrifices that are necessary to obtain it - after all, the funds used for healthcare cannot be allocated to alternative uses. Affordability is therefore an important consideration that is closely linked to access. Quality determines the health value of the treatment provided. Arriving at a social consensus on how to achieve these goals is difficult, however, which in most countries leads to intense debate on healthcare, as the contributions to this book regarding the US, South Africa, Colombia and the Netherlands all illustrate. Unsurprisingly, there is no one particular healthcare system that meets all three of the needs identified above perfectly. Instead, there is a wide variety of such systems, each with different advantages, disadvantages and trade-offs. Hence it is important that data on the problems encountered are collected and analysed, and that learning occurs between different health systems. This is a practical as well as a scientific challenge, because hitherto most studies on healthcare regulation have not taken a comparative perspective based on comparable data. In fact, in many respects, no such data yet exists.

This book charts hospital financing across the three dimensions of access, affordability and quality. It does so based on an international comparison spanning four different continents. For the purpose of our project, we have collected 11 country reports, compiled by national experts according to a standard structure. In addition, six thematic chapters are included that explore specific questions. The invited authors include academics and practitioners (primarily, but not exclusively, policymakers).

We have focused on hospital financing for the following reasons. 
Hospital care is in most countries responsible for the main part of (curative) health care expenditure. As a result of this, it is also the most intensively studied part of health care, which facilitated both the collecting of comparable data and provided a basis for discussion between experts from different countries. As for practical considerations, covering the entire field of health care services would have resulted either in a more superficial approach or in an even larger volume.

We have worked closely together with the authors in order to provide a common starting point and to refine the standard structure. This international cooperation will hopefully also contribute to creating a network on these issues that will outlive the process of writing this book. No such group currently exists in the OECD context for healthcare services. But in fact, we have been (at least somewhat) more inclusive than the OECD is today with contributions from South America and Africa alongside North America and Europe - although for a variety of largely practical reasons (such as distance and pre-existing personal networks), the majority of the contributions collected regard EU Member States. Our target audience of both practitioners and academics is not just multinational but multidisciplinary, covering law, economics, health sciences and public administration.

We hope that the publication of this book will enrich the available literature on healthcare financing. Earlier publications on healthcare financing have been different in their approach. For example, Paolucci ${ }^{1}$ focuses on insurance design and the distinctions between mandatory versus voluntary forms of insurance and basic versus supplementary coverage. The OECD presents various documents (e.g., Healthcare Systems, Efficiency and Policy Settings) ${ }^{2}$ containing quantitative information about the healthcare systems of different countries and there is some categorization of their healthcare systems. Britnell ${ }^{3}$ provides a useful but non-systematic description of many healthcare systems around the globe. The main methodological innovation of this book is that it uses country reports based on a standardized structure to facilitate cross-comparison, alongside thematic chapters and an attempt at synthesis in the introduction. This resembles the approach to healthcare fraud and corruption adopted in Mikkers, Sauter, Vincke and Boertjens, but is more ambitious in subject, geographic scope and depth. ${ }^{4}$

1 Francesco Paolucci, Healthcare Financing and Insurance: Options for Design (Developments in Health Economics and Public Policy), Springer, 2010.

2 http://www.oecd.org/eco/healthcaresystemsefficiencyandpolicysettings.htm, last accessed 29 January 2019.

3 Mark Britnell, In Search of the Perfect Health System, Palgrave, Macmillan Education, 2015.

4 Misja Mikkers, Wolf Sauter, Paul Vincke and Jos Boertjens (eds), Healthcare 


\section{RESEARCH THEMES AND QUESTIONS}

We did not set formal research questions for our authors or indeed for ourselves, but in the course of our exchange some of the following core themes emerged, which can be framed as research questions:

- What are the main forms of hospital financing?

- What are the main challenges to the financing of healthcare systems?

- Are there essential differences here between tax-based and insurance-based systems?

- Can we relate financial inputs to health outcomes at the national level in a meaningful way?

- What do we need in terms of information in order to compare the performance of healthcare systems effectively?

In all cases, of course, the dimensions of access, affordability and quality are essential. The particular final challenge that we set ourselves is whether it is already possible to define best practices.

In an attempt to provide at least a partial answer to some if not most of these questions, we will now discuss each in turn: trends in expenditure, the relevance of market structure, payment models, access and waiting times, entitlements and gatekeeping, and finally the regulatory landscape. More granular answers are, of course, to be found in the individual chapters that follow below.

\section{Trends}

First, we will present some insights regarding the performance of hospital systems in different countries. ${ }^{5}$ This is no easy task. The performance of healthcare systems is inherently difficult to measure - particularly in terms of outcomes. This holds a fortiori for hospital performance. Data on hospital costs, hospital outcomes and access to hospital services for different groups in societies are not only relatively scarce, but even more difficult to compare. We appeal to policymakers in different countries to focus on

Fraud, Corruption and Waste in Europe. National and academic perspectives, Eleven International Publishing, 2017.

5 The data used in this paragraph are downloaded from the OECD health data base. (https://stats.oecd.org/index.aspx?DataSetCode=HEALTH_STAT, last accessed 29 January 2019). The data, figures (and additional figures not presented in this chapters) and the code to produce the figures can be found on: https://github. com/misjamikkers, last accessed 29 January 2019. 


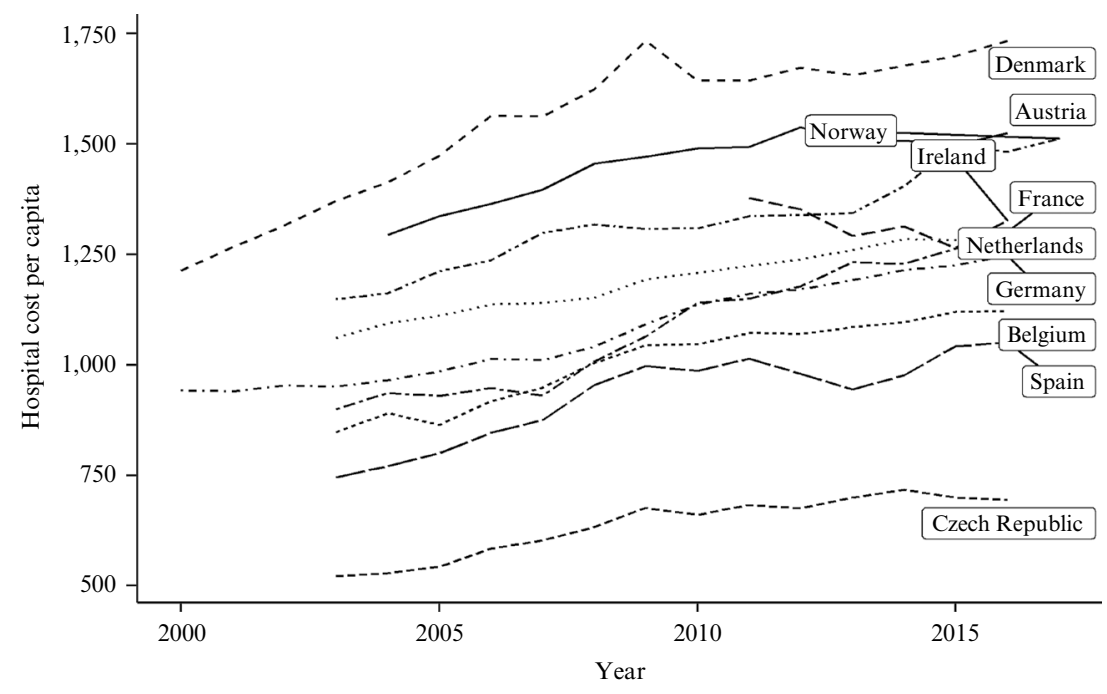

Figure I.1 Hospital costs over time

data collection and to agree on relevant criteria, so that meaningful crosscountry comparisons are easier to make. This will facilitate learning from international experiences and would, we believe, be a relatively low-cost and low-risk way of improving outcomes more quickly and effectively.

The countries that we have examined all have different levels of hospital spending, but seem to be subject to the same trends. The overall trend in all the countries presented in the book and present in the data is that they are spending more money on hospital services ${ }^{6}$ over time, Figure I.1. However, the worldwide financial crisis may have slowed the growth of hospital expenditure.

One key reason why the cost of hospitals is increasing is that medical technology is advancing. In Figure I.2, we present the growth in MRI scanners as an example. ${ }^{7}$

All the countries observed, with the exception of Ireland, have seen a trend towards higher spending on hospitals and an increasing number of MRI scanners per million inhabitants.

In general terms, technology is becoming a more important element of hospital treatments, while the time spent in hospitals ('the length of stay')

6 Cost is presented in terms of cost per capita in constant PPP's.

7 https://github.com/misjamikkers, last accessed 29 January 2019. Contains also examples of MRI and PET scanners. 


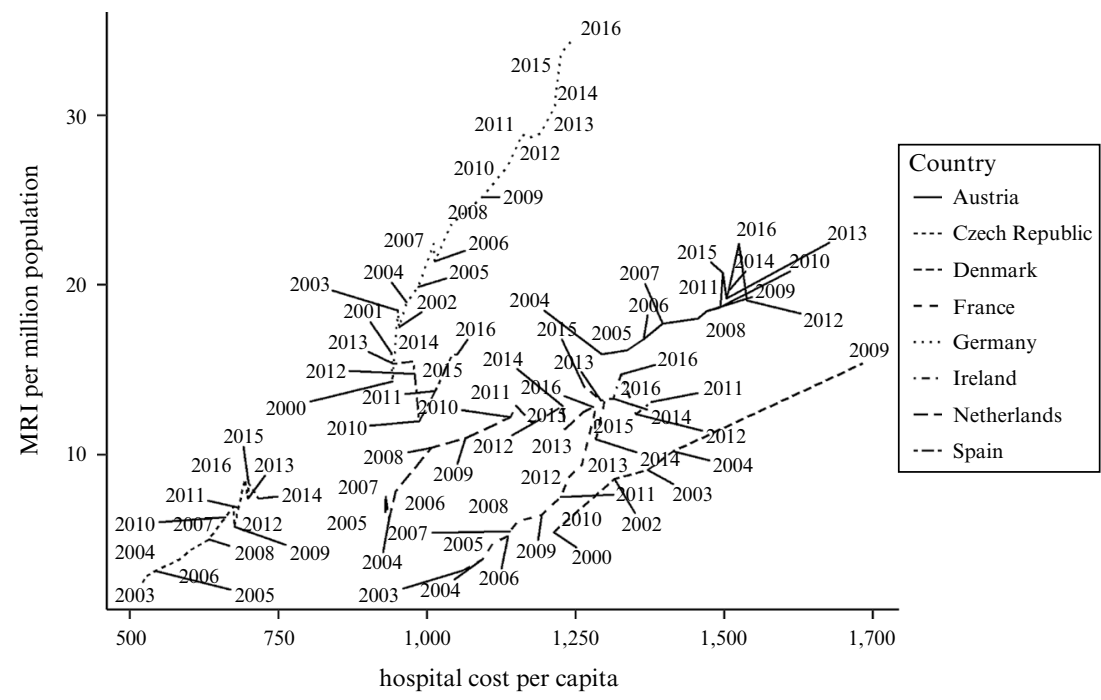

Figure I.2 Hospital costs and the number of MRI scanners per million of population over time

is decreasing in all countries. ${ }^{8}$ The increasing use of technology has an effect on hospital outcomes such as 30-day mortality after admission to hospital for acute myocardial infarction (AMI), as shown in Figure I.3 and - although less pronounced - in an increase in the five-year net survival rate for breast cancer.

The most important conclusion of this section is that while countries differ at the level of available indicators, they are exposed to similar trends. Hence there is scope to learn from different hospital financing systems and the organization of hospitals in different countries.

\section{Market Structure}

In all the countries examined, people have freedom of choice when it comes to hospitals, and hospitals are paid in relation to the number of patients treated (and/or the amount of treatment provided). These two elements mean that hospitals compete with each other to some extent. In countries like the Netherlands and Colombia, hospitals compete on the

8 See for data and figures: https://github.com/misjamikkers, last accessed 29 January 2019. 


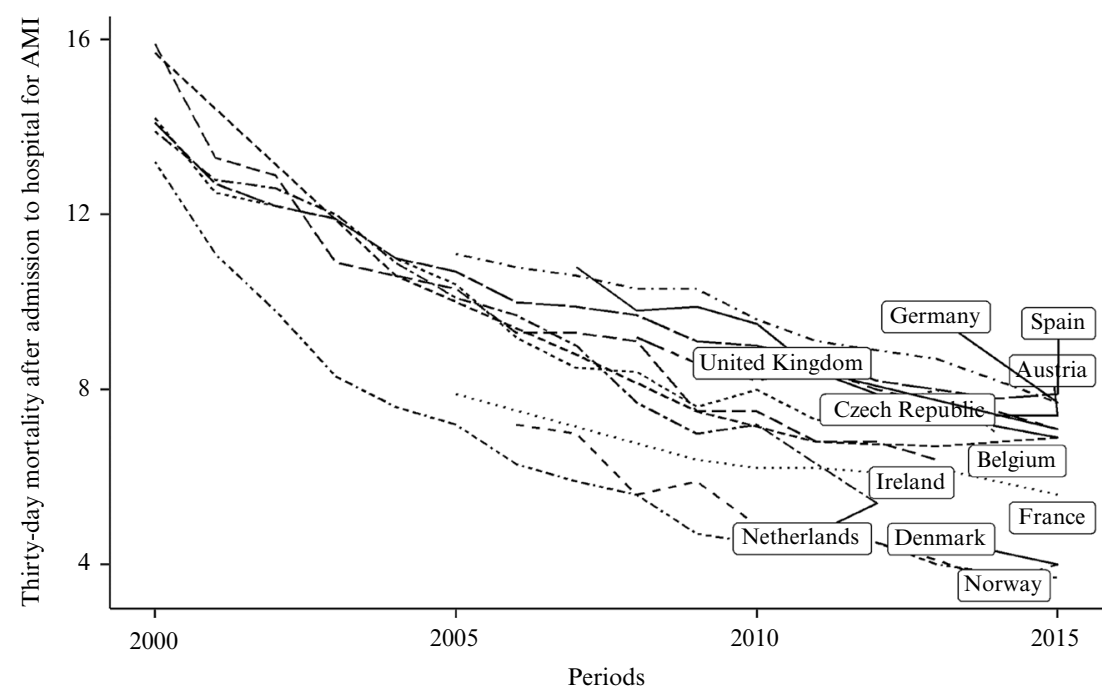

Figure I.3 Mortality following an acute myocardial infarction

dimensions of price and quality, while in most other countries prices are regulated by the governments and therefore hospitals compete primarily on the quality dimension. ${ }^{9}$

Evidently, market structure is neither immutable nor uncontested. In many countries, including the Netherlands, France, the UK and Belgium, policymakers are currently debating topics such as mergers between hospitals and the optimum size for hospitals (with respect to quality).

As shown in Figure I.4, the size of hospitals (measured in beds per hospital) differs from country to country. Most countries are seeing an increase in the scale of hospitals over time (again with the notable exception of Ireland), although the number of beds per million inhabitants is declining. ${ }^{10}$ This means that the increase in scale must be attributable either to mergers or the closure of hospitals.

Obviously, the countries presented in the book differ greatly in terms of population, income, population density and in many other respects. These differences probably play a role in the size of hospitals. Figure I.5

9 See e.g., Martin Gaynor, Rodrigo Moreno-Serra, and Carol Propper, 'Death by Market Power: Reform, Competition, and Patient Outcomes in the National Health Service' (2013) 5(4) American Economic Journal: Economic Policy 134-66.

10 See https://github.com/misjamikkers, last accessed 29 January 2019. 


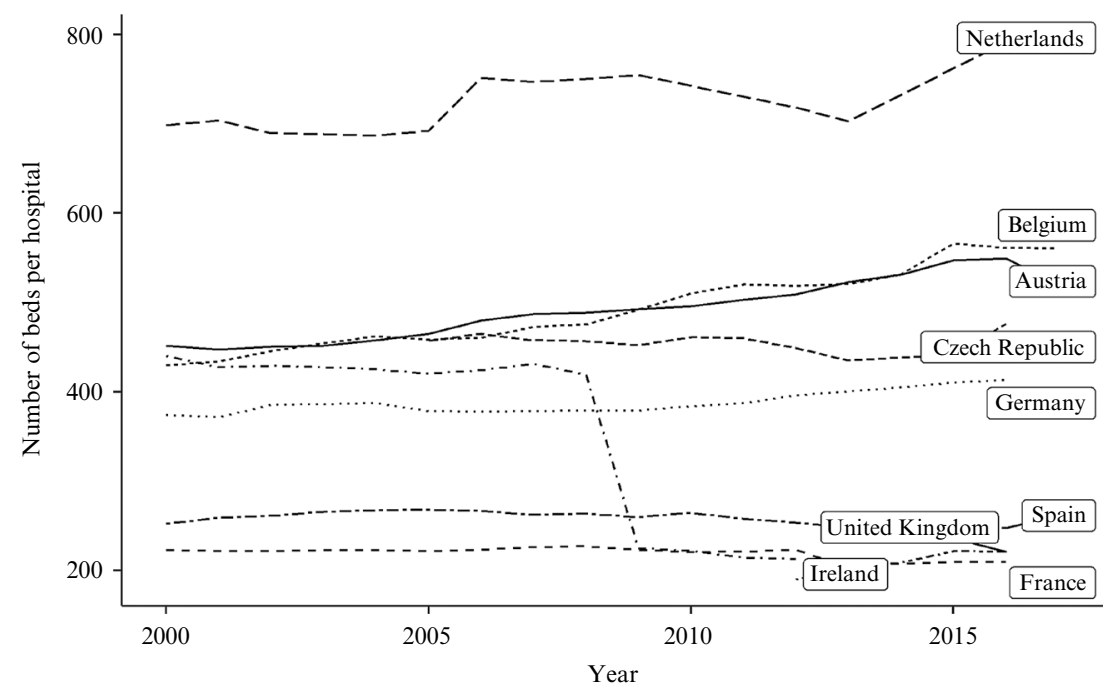

Figure I.4 Hospital size over time

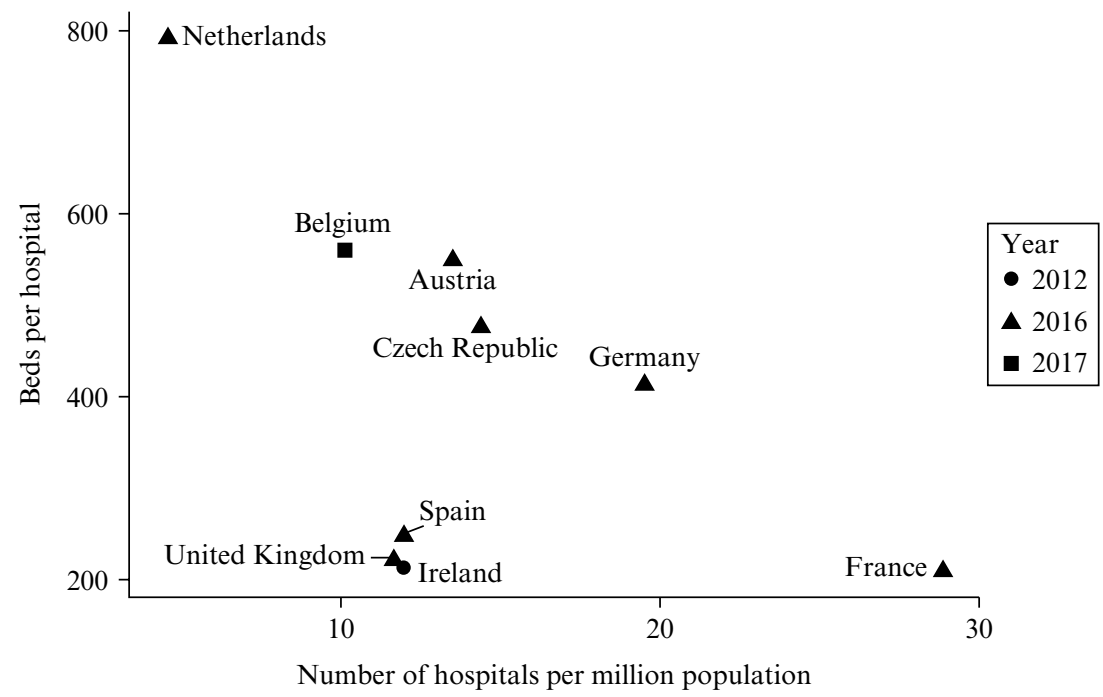

Figure I.5 Trade-off between the number of hospitals (per million inhabitants) and the size of hospitals 
shows - based on the last data available - a trade-off between the number of hospitals (per million inhabitants) and the size of hospitals.

The relationship between country size and hospital size is less obvious. However, the existence of fewer hospitals per head of population logically means that hospitals tend to be larger. While the Netherlands has a smaller number of very large hospitals, France has many smaller hospitals. The UK, Spain and Ireland have a relatively small number of small hospitals. Dutch hospitals are nearly four times larger than French hospitals.

\section{Reimbursement Models}

The country reports reveal a wide variety of hospital financing models. In most countries, some form of diagnosis related group (DRG) financing model is in use, combined variously with a fee-for-service models for specialists, block grants, direct public financing and separate financial arrangements for specialized services and/or infrastructure.

However, payment models cannot be viewed separately from some of the major issues that we have tried to address in this book: accessibility and affordability. In Figure I.6, we illustrate this by showing some of the most important financial incentives associated with various types of financing.

All countries are struggling to contain steadily rising expenditure and, at one stage or another, all the models presented in the various chapters were an attempt to regulate either prices or total expenditure.

Figure I.6 illustrates that the incentive for providers to control costs is weakest under a fee-for-service system and probably strongest under a capitation model. DRG-type compensation and fee-for-service models have the disadvantage of incentivizing volume. In countries where the costs

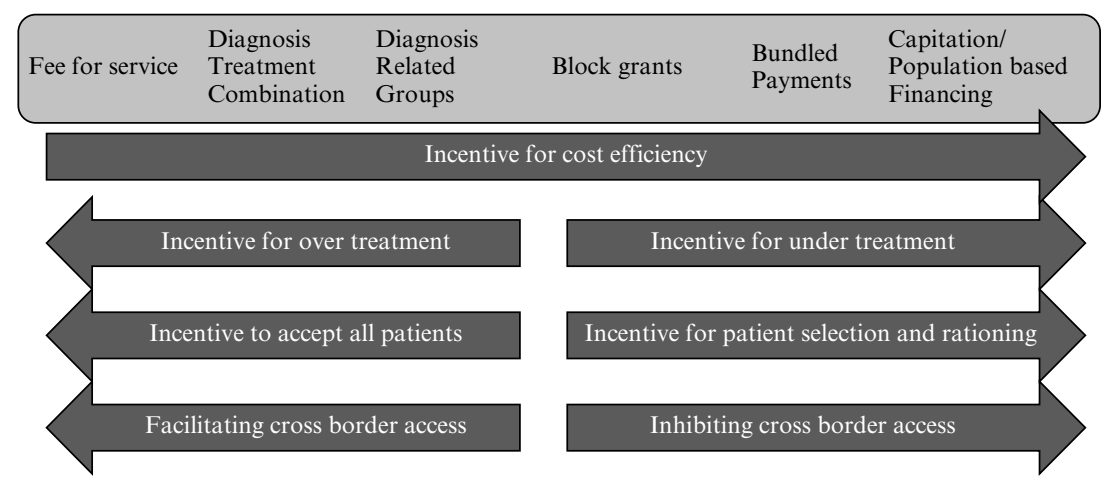

Figure I.6 Payment structure and incentives 
of capital are also covered by DRG-type payments, this incentive is even stronger because marginal revenue will always exceed marginal costs. This may easily lead to overtreatment or waste within the system. In his contribution to this book, Sage notes that the US healthcare system manages to generate annual waste estimated to amount to $\$ 1,500,000,000,000 .{ }^{11}$ Despite this obvious disadvantage, DRG-type financing is the most widespread reimbursement model. At the same time, volume-dependent financing fails to offer an adequate basis for the provision of services in sparsely populated areas, where regional or national government usually has to intervene to maintain an adequate level of services. We see this type of intervention in several countries (notably in France, Colombia, and Germany).

Where payment models for hospital care are primarily developed to enhance cost-efficiency, they also have to meet the other policy targets: accessibility and quality of care. Figure I.6 shows that, theoretically, the fee-for-service and DRG type models incentivize providers to accept new patients (provided that on average the fee per patient covers the costs). In a system with these types of payments, you would not expect long waiting lists, unless there is a capacity constraint (such as limits on market entrance or personnel available). In the Netherlands, for example, a block grant model/population-based financing system was replaced with the DTC system precisely to address growing waiting lists (among other issues). The UK, Czech Republic and Ireland chapters also describe moves away from the block grant system and towards more activity-based and/or quality-based payments.

An interesting angle of the relationship between payment system and accessibility is explored in the chapter by Burke on cross-border healthcare. To facilitate cross-border healthcare, it is necessary to calculate costs per patient; countries with a fee-for-service or DRG-type system are simply better equipped to calculate these costs, so we would expect the threshold for accepting and treating patients from other Member States (or from outside the EU and its affiliate countries) to be lower.

All the countries covered in this book are struggling to balance costcontainment with access to healthcare. In several countries (such as England and Norway), there seems to be a tendency to turn away from reimbursement models that include an incentive to generate volume and move towards models that emphasize quality of care and/or 'value' (although in other countries such as Ireland, Belgium and the Czech Republic, no such trend is evident). Janus describes an experiment in New

11 See page 24 . 
York state, whereby the funding model is slowly being transformed from an FFS/DRG model to one that combines bundled services and capitation.

If one of the main disadvantages of DRG-type or fee-for-service payments is the tendency to incentivize volume, another drawback is that they generally do not differentiate on quality or outcome of care. This is despite the fact that overtreatment can be just as detrimental to patients' welfare as undertreatment. In several countries, we see a tendency to experiment with payment models to incentivize quality or the value of care instead of maximizing volume. The UK is using the concept of payment by results and in the US, for example, a fairly large-scale experiment was carried out in New York state with an alternative model, the Delivery System Reform Incentive Payment Program, as described in the chapter by Janus. This reform has a triple aim: better health, better care and lower cost, which sounds like every healthcare policymaker's dream. A theoretical approach to a population-based reimbursement model is offered by Bogetoft et al. Several experiments are underway with this type of contracting. What all these models have in common, is that the healthcare provider is a risk bearer when it comes to the costs of healthcare, but can also benefit from improving the health status of its allocated population.

From the contributions to this book, we can conclude that most countries are facing some common problems in healthcare financing: rising costs (due to ageing populations and technological improvements), guaranteeing access and improving the efficiency and quality of services. As far as payment systems go, we see that these systems have to address these - sometimes conflicting - policy aims simultaneously, which probably accounts for the wide variety of systems and apparently random modification of systems that we see. If the holy grail of healthcare financing exists - a form of financing that addresses all three of these problems simultaneously and also achieves the triple objectives of better care, better health and lower costs - it remains well hidden. However, this does not mean we should stop searching. These topics define the stakes for hospitals, insurers, policymakers, patients and tax paying citizens. Hospitals in the light of rising costs, will probably face new forms of regulation with an aim to curb their revenue and the others, if these measures fail, will have to foot the bill, either through restrictions in access or by rising taxes and premiums.

\section{Accessibility of Healthcare}

Regarding the accessibility of hospital care, three constituent elements can be highlighted: first, who is responsible for guaranteeing accessibility; secondly, standards and outcomes in relation to waiting lists; and thirdly, the extent of private or out-of-pocket payments. 
Regarding responsibility, two types of systems have traditionally been distinguished: a system in which healthcare is funded directly by the State from taxes (the Beveridge model) and a system in which funding is provided by healthcare insurers (or funds), which are in turn financed by contributions from affiliates (the Bismarck model). As a consequence, responsibility for guaranteeing accessibility to healthcare facilities resides - generally speaking - with the State or the healthcare funds, respectively. In some cases, general norms exist regarding accessibility, in the form of governmental guidelines. But these do not lead to the existence, under Beveridge-type systems, of an enforceable individual right to (accessible) healthcare for citizens. In the case of healthcare funds or insurers, the infringement of accessibility norms may lead to individual claims by insured on the basis of non-compliance, as the example of Colombia shows. Additionally, insurers may be subject to legal obligations regarding access to healthcare, as is the case in the Netherlands, and risk government sanctions in cases of non-compliance.

Turning to the facts on the ground in relation to accessibility, a distinction can be made between dimensions of time and space. The maps showing the geographical distribution of hospitals included in the country reports, together with the tables on waiting times for hospital care, illustrate that notable differences exist not just between countries but also within countries. This finding puts the comparability of data between countries into perspective. One explanation for the lengthening of waiting lists at the national level is provided by the theoretical model developed by Rodríguez-Álvarez and Perez-Villadoniga in their chapter on the determinants of waiting lists in Spain. Although technological progress may help to reduce waiting lists, it may also mean that previously terminal illnesses become chronic conditions, thus increasing life expectancy but also morbidity, chronicity and overall demand for care.

Regarding binding norms on waiting times, a picture emerges in which some countries have established norms (including England and the Netherlands), while in other countries no such norms exist (in Germany and South Africa, for varying reasons), and in a third group norms are expected to be developed (Austria).

Another factor is that information on waiting times varies greatly between countries. As Burke demonstrates in his chapter, the EU Cross Border Healthcare Directive, due to the lack of concrete norms, does not require Member States to further provide relevant information in this regard. In addition, the rights bestowed are limited by various factors most importantly, a lack of information on quality, safety and prices, all of which would be necessary to assess whether treatment abroad is indeed quicker, safer or more expensive than similar treatment at home. 
Solutions to the problem of accessibility, as Burke demonstrates, are not be expected from the Cross Border Healthcare Directive in the short term, since the necessary conditions for operationalizing (in addition to activating) the aims of the directive, notably in the form of intermediaries, are currently lacking.

A further restriction on accessibility to healthcare services (including hospital care) may be imposed by the obligation to see a general practitioner before hospital care can be provided. The country reports demonstrate that in most countries this is formally the case. However, in practice outcomes may differ since in some countries this is considered to be a breach of freedom of choice in healthcare, and legal recourse to enforce this right may be available.

Access to healthcare services may also be limited by the existence of private payments and deductibles. Most frequently, private payments concern out-of-pocket payments, where a fixed amount is paid by the user for each service. These amounts may vary according to individual differences (such as a patient's income) or - where applicable - on the basis of a person's individual choices (in relation to an insurance policy).

In sum, while accessibility to healthcare can be measured in terms of waiting times and distance, there may be other restrictions that are more difficult to compare as they reflect the particular characteristics of a given healthcare system.

\section{Regulatory Framework}

Although the country chapters do not focus on legal frameworks, these do form an important contextual variable. There are at least three different dimensions by which the relevant regulatory frameworks that are covered in this book can be distinguished. However, it is not - or not yet - possible to identify a strong correlation between these characteristics and health outcomes.

- The first type is the regulatory framework for funding. Most countries have either a predominantly tax-funded or a mandatory insurance-funded system (sometimes based on employment status). In practically all cases, there is also private funding for alternative or supplementary care, which may be based on voluntary insurance (as is the case in Ireland and the Netherlands for instance). In addition, private (co-)payments may be involved. This variety indicates that while regulatory regimes are often clear when observed from a distance, there tends to be complexity, with cross-cutting rules, upon closer observation. 
- The second dimension is that of the level of governance, with varying degrees of centralization or decentralization. The four different countries that constitute the UK each have their own national health services that combine governance and provision. In federal systems, such as Austria, Belgium and Germany, significant regulatory responsibilities for healthcare accrue at the regional state level. However, even in unitary states such as France and South Africa, significant regulatory powers with regard to healthcare are decentralized to a significant degree to the regional or provincial (or even local) level. In the Netherlands, private providers and insurers are seen as the central actors, with market-based governance of both in the background, and centralization is more pronounced with independent administrative authorities playing a significant regulatory role. In general terms, decentralization may enhance legitimacy and therefore responsiveness compared to tighter centralized (budgetary) control.

- The third relevant dimension of regulation is that of healthcare provision and how it is assured. Here, the basic distinction with regard to regulation is that between public and private ownership of healthcare providers. There is a difference between systems where regulation is used to direct the conduct of private undertakings in a market context (healthcare providers and/or insurers) and systems with public provision where regulation takes place within a vertically integrated column of command and control, or at least of internal instructions. As mentioned, most countries observed rely on a mixture of such public and private elements.

The resulting complexity makes it difficult to see which regulatory elements, if any, contribute the most in terms of quality, access and affordability. Variables such as waiting times and shortages, as cited in the previous section, can only provide a partial indication. Arguably, marketbased systems are capable of reacting more quickly to variations in supply and demand, but structural and distributional problems may still occur where incentives are not fully aligned or where market power can occur in the absence of an effective public framework. On the other hand, and from a more principled or ideological perspective, public provision may in any event be viewed as more easily compatible with democratic control and consequent willingness to pay than private provision. However, we have found no clear results on this dimension, and it is not obvious that a more refined comparative exercise would have merit.

As the example of the US (among others), as discussed by Sage, demonstrates, the scope for debate on the need for healthcare regulation 
and how to calibrate it is essentially open-ended and such debate is not necessarily productive. Where there is significant tension between supply and unmet demand within a public healthcare system, both polarization and flight from the public system may take place, as is illustrated by the case of South Africa. Norm-based regulation which incorporates standards of fairness such as a duty of care, while at the same time enabling transparent budget checks, would appear to be a central requirement. Once such norms have been accepted, it may be useful to depoliticize their administration - whether this occurs primarily through the courts as in Colombia, or through specialized administrative authorities as in the Netherlands and the UK.

Given the diversity of national settings, it is unrealistic for us to derive any recommendations for regulatory design beyond the most general ones from the material we have collected so far. However, the chapters by Burke, Sage, and Boertjens and Guy provide more specific discussions of the legal and regulatory dimension for several specific jurisdictions: the EU (although it does not formally regulate its own healthcare system, as such), the US, and the cross-country comparison between England and the Netherlands.

\section{ACKNOWLEDGEMENTS}

First of all, we would like to thank all the authors for their contributions: it has been a genuine pleasure to work with all of you, even if we required repeated changes in format and presentation in order to facilitate comparison. In the course of writing and editing this book we have enjoyed frequent interaction and discussions with all of you which was very helpful in identifying common themes and approaches. This in turn has led to further mutual contact, including with regard to new projects outside the scope of the present venture. We hope and trust this collaboration will continue.

Secondly, we would like to thank Brenda Mook and Aad Noordermeer for their invaluable support in all organizational matters and Toby Adams for his language support. Mireille Coebergh and Karlijn Bokkers provided welcome and timely editorial assistance. We are very grateful to TILEC (Tilburg University) and the Dutch Healthcare Authority (NZa) for funding this project. We would like to thank the Chairwoman of the NZa, Marian Kaljouw, and the Director of Healthcare Regulation, Josefien Kursten, for graciously supporting the project in various other ways as well.

Thirdly, by way of a friendly exhortation rather than an acknowledgement, we would like to make an appeal to our readers. In many ways, this 
project remains incomplete: much remains to be learned regarding the comparative study of healthcare financing and our contribution remains a modest beginning. Nevertheless, we hope that this effort will encourage readers to take up further research in this direction.

Finally we have been extremely lucky with the editorial team at Edward Elgar. We would like to thank Luke Adams, Jennifer Lee, Laura Mann and Stephanie Tytherleigh as well as their colleagues with whom we have not had direct contact but who have worked behind the scenes on a highquality production. They have enabled us to complete the book as we had imagined it - and we are in any event very happy with the result.

We dedicate this book to all its contributors and to the spirit of international collaboration and inquiry in comparative healthcare financing research. 